\title{
L’apport de l'imagerie à la neuropsychologie
}

\section{The contribution of imaging to neuropsychology}

\author{
Adrien Folville ${ }^{1,2}$ \& Jonathan Fortier ${ }^{3}$ \\ ${ }^{1}$ GIGA-CRC In Vivo Imaging, Université de Liège, Belgique \\ ${ }^{2}$ Département de Psychologie, Unité de Recherche de Psychologie et de Neurosciences de la \\ Cognition, Université de Liège, Belgique
}

${ }^{3}$ Laboratoire de psychologie des Pays de la Loire (EA 4638), Université d'Angers, Maison de la recherche Germaine Tillion, Angers, France

Auteurs correspondants :

Adrien Folville: adrien.folville@uliege.be

Jonathan Fortier: jonathan.fortier@univ-angers.fr

\section{Résumé}

La neuroimagerie (IRM, TEP, EEG et MEG) est utilisée au sein de la communauté scientifique depuis plusieurs décennies. Outre son aspect purement médical, elle est devenue une approche complémentaire à bien des domaines, comme c'est le cas pour la neuropsychologie. Toutefois, depuis son apparition, l'apport réel de l'imagerie à la compréhension du comportement humain fait débat dans notre domaine. Afin de pouvoir permettre une réflexion commune sur la thématique, la Société de Neuropsychologie de Langue Française (SNLF) a organisé un débat entre les professeurs Bernard Laurent (Saint-Etienne) et Martial Van der Linden (Liège \& Genève). Chacun à leur tour, les deux orateurs ont exposé leur vision de l'apport de l'imagerie à la recherche ainsi qu'à la clinique en neuropsychologie avant de laisser place à une interaction avec le public. Cet article reprend les différents points abordés tout au long de ce débat ainsi que les diverses interventions.

Mots-clés : neuropsychologie, neuroimagerie, cognition 


\section{Abstract}

Neuroimaging tools (MRI, PET, EEG and MEG) have been widely used within scientific community for more than four decades. Beyond its medical use, imagery has become a complementary approach to many research areas, as it is the case for neuropsychology. Nowadays, however, whether imagery has been contributing to our understanding of how human's mind generates behaviours and thoughts remains under debate. Notably, it has been argued that neuroimaging (e.g. $\mathrm{fMRI}$ ) provided insights about the brain localisation of human behavioural processes rather than informing into how these processes were implemented. On December $1^{\text {th }}$ of 2017, the French language Society of Neuropsychology organised a debate with Bernard Laurent (Saint-Etienne) and Martial Van der Linden (Liège \& Genève) that aimed at shedding light on this controversial topic. The debate was based on a list of items to be discussed. Through an overview of his own researches, each speaker discussed on the relevance of using neuroimaging as a mean to understand origins of our behaviour. Bernard Laurent explained how imagery tools have grown up our theoretical and clinical knowledge of cognition. In contrast, some limitations of the current use of neuroimaging were noted by Martial Van der Linden. This article sums up the points raised in the debate.

Key-words: neuropsychology, neuroimaging, cognition

\section{Introduction}

Afin d'alimenter ce débat sur l'apport de l'imagerie à la neuropsychologie, Didier Le Gall (Angers), animateur de la session, a rappelé, en quelques grandes dates et quelques images, l'évolution rapide des techniques et leur expansion vers des champs d'application toujours plus diversifiés. De cette rapide introduction, un certain nombre de questions se posent qui sont adressées aux deux orateurs :

Où en sommes-nous vis-à-vis des problèmes méthodologiques rencontrés dans les études en imagerie : activation/inhibition ; soustraction ; traitement de données ?

Les avancées technologiques en imagerie cérébrale sont-elles bien exploitées et sont-elles toutes exploitables?

Existe-t-il une comparabilité et une complémentarité utiles des données entre imageries morphologiques et fonctionnelles?

L'imagerie cérébrale fera-t-elle un jour le diagnostic en lieu et place de l'analyse clinique ? 
Est-ce qu'en regard des données disponibles en imagerie cérébrale, la notion de centre d'activité, telle qu'elle est encore largement utilisée en clinique quotidienne, garde sa pertinence pour rendre compte de ce que nous observons chez les patients?

Quelles sont les postes de coopération entre IRMf et EEG ou MEG ?

Toutes les études en imagerie sont-elles, par définition, pertinentes ? Quelles sont les grandes évolutions à venir ?

\section{La révolution de l'imagerie médicale.}

II y a maintenant près de 50 ans, lorsqu'un étudiant de médecine rentrait en internat, on lui apprenait qu'il était impossible de faire de neurologie sans connaitre les grandes coupes cérébrales. Les internes passaient donc des heures à regarder et à apprendre ces grandes coupes. Cependant, en 1978, l'apparition des scanners dans les hôpitaux créa une première révolution. Cette avancée technologique entraina un véritable bouleversement dans les études menées qui permit un grand nombre de recherches encore très connues aujourd'hui, comme par exemple les études de Bernard Laurent sur l'infarctus thalamique ou l'amnésie thalamique $[1,2]$.

\section{L'imagerie permet-elle d'aborder le cas unique?}

Certains chercheurs pensent que l'une des limites de l'imagerie cérébrale pourrait être l'impossibilité de l'appliquer à l'étude du cas unique. Prenons l'exemple du cas HM [3]. Grace à sa conservation du gyrus para-hippocampique droit, HM garde une mémoire visuelle de ses nouveaux appartements. II conserve également des capacités d'acquisition visuelle. Aujourd'hui, il est possible de réaliser de l'imagerie de manière individuelle, une partie des critiques à l'encontre l'imagerie tombe de ce fait. Cependant, selon Bernard Laurent, il serait indispensable d'avoir de bons protocoles pour faire de l'imagerie, comme par exemple les 
études de Dehaene sur les bases neuroanatomiques de la lecture [4]. Ces paradigmes se basent en effet sur des hypothèses et sur des processus neurocognitifs connus.

\section{Exemple d'utilisation de protocoles de neuroimagerie pour l'étude de la douleur}

Depuis les années 1990, il a été montré que la douleur appartient aux champs de la cognition et de l'émotion. Au début de ces recherches, la douleur a été envisagée sous l'angle de sa matrice, en croyant trouver le graal de l'intégration douloureuse. Ensuite, les recherches se sont intéressées au cortex de modulation (cingulaire antérieur et orbitofrontal). Ces zones cérébrales se sont avérées peu spécifiques, sans contrôle des endorphines, ce qui a poussé les chercheurs à se tourner vers le marquage des endorphines. Selon Bernard Laurent, voir une personne souffrir ou expérimenter soi-même la douleur activerait les mêmes zones cérébrales, à quelques nuances près.

Cependant, la question de l'utilité pour les patients demeure. C'est par exemple le cas dans la maladie d'Alzheimer. Dans cette pathologie, les patients ont des difficultés de restitution ou d'exagération de la douleur. Par exemple, l'équipe de Bernard Laurent pensait que certains patients étaient presbyphagiques, alors qu'en réalité une grande partie des réponses était exagérée. Cette même équipe s'est également intéressée à la fibromyalgie ainsi qu'à d'autres maladies somatoformes pour en trouver les signatures nociceptives. Ils se sont très vite rendu compte que le fait d'induire une douleur physique ou de feindre l'induction d'une douleur (par hypnose) ou même d'évoquer une douleur avec un patient activerait les mêmes zones cérébrales. Il semblerait donc que faire ressentir et imaginer une douleur implique un même réseau localisé dans les régions insulaires antérieures. Si les entrées sensorielles étaient différentes (nociceptive, auditive, visuelle...), l'imagerie devrait nous permettre de voir l'aire corticale associée. Cependant, le reste du circuit d'analyse de l'information (aires multimodales) semble rester le même, quelle que soit l'entrée sensorielle.

\section{Limites de l'imagerie}


Grace à l'imagerie il serait donc uniquement possible de connaitre l'entrée sensorielle mais il serait impossible de savoir ce qu'il se passe dans les matrices plus élaborées.

Par exemple, en imagerie fonctionnelle, le signal BOLD (blood-oxygen-level dependent) illustre les changements d'oxygénation des zones cérébrales dus à une activité. Or ces changements ne sont visibles que plusieurs secondes après la réponse neuronale. Ces quelques secondes représentent un temps énorme pour les neurophysiologistes. Bernard Laurent illustre ainsi l'interprétation du signal BOLD comme étant une table desservie après un repas sur laquelle nous essayerions de reconstituer la conversation s'y étant tenue. Pour illustrer à quel point ce décalage temporel est important, il est possible de prendre l'exemple d'enregistrements intracérébraux chez des patients épileptiques. Le temps de réaction des patients à la douleur est d'environ 200 millisecondes. Ceci illustre donc bien à quel point les techniques d'imagerie ont encore besoin de perfectionnement pour capter les subtilités des réactions neuronales.

Les travaux de Bernard Laurent sur la douleur peuvent être mis en lien avec les recherches de certains chercheurs travaillant sur des thématiques proches. C'est par exemple le cas d’Emmanuel Barbeau qui étudie la reconnaissance visuelle avec le DMS 48 [5]. Selon lui, les régions cérébrales impliquées dans ce type de tâche sont principalement l'amygdale, l'hippocampe et le gyrus cingulaire antérieur. Ce sont donc les mêmes zones que celles incriminées dans les études de nociception de Bernard Laurent. Nous constatons ainsi que, quelle que soit la modalité d'entrée, le même réseau de traitement semble ensuite s'activer. La grande question qui subsiste est de savoir comment ce réseau va se distribuer ? Qui va décider que le signal s'arrête à l'amygdale et continue ensuite jusqu'à l'hippocampe ? Selon le modèle de Fauchon et al 2017 [6], si nous considérons toutes les zones où nous savons que le message sensoriel arrive, grâce à la connectivité fonctionnelle, nous devrions pouvoir essayer d'établir un modèle. Grâce à cela, nous pourrions déterminer où interviennent le contrôle inhibiteur ainsi que l'intégration du contexte empathique.

Potentielles dérives de l'utilisation de l'imagerie 
D’autres études évaluant la connectivité cérébrale en fonction du genre ont été réalisées [7]. La connectivité intra-hémisphérique serait plus importante chez l'homme alors qu'à l'opposé, la connectivité inter-hémisphérique (principalement antérieure) serait plus importante chez la femme. Il semble légitime de se demander quel est l'intérêt de ce genre de résultat ? Vientil d'une différence culturelle, de l'épigénétique ou d'une différence acquise? Dans cette même étude [7], un groupe d'enfants de 8 ans était testé et la même différence de genre a été observée. Cette différence en fonction du sexe pourrait donc être d’origine épigénétique, ce qui n'est pas sans intérêt.

Enfin, il convient d'évoquer d'autres dérapages possibles associés à l'utilisation de l'imagerie cérébrale. Une étude [8] a, par exemple, cherché à étudier les différences de douleur ressentie entre des croyants (chrétiens) et des non croyants lorsqu'ils regardaient une image de la vierge Marie. Les auteurs ont analysé les différences en terme de réponse, y compris sur la sécrétion d'endorphines. Une dernière étude [9] a également exploré le désir sexuel féminin lors du visionnage d'images érotiques et non érotiques. Selon les auteurs, les femmes se plaignant d'hypoactivité sexuelle par manque de désir auraient un gros effet d'ordre social ou de fonctions cognitives ainsi qu'un plus fort effet des zones inhibitrices que la moyenne. Ce type de résultat est non seulement discutable d'un point de vue théorique mais est également d'un intérêt relativement limité.

\section{La perte d'influence des modèles psychologiques}

Bien qu'il ait pris part à la mise en place d'études en neuroimagerie durant sa carrière, Martial Van der Linden s'est progressivement centré sur une remise en question du réductionnisme en psychopathologie [10]. II se désintéresse ainsi progressivement de la neuroimagerie afin de dénoncer les dérives pouvant être associées à l'utilisation en clinique du DSM V ainsi qu'au concept de "maladie d'Alzheimer ». II dénonce également une perte de légitimité et d'influence du niveau d'analyse psychologique au profit d'une neuroculture réductionniste 
[10].

Plusieurs raisons ont été évoquées pour expliquer ce désintérêt grandissant pour l'étude des modèles psychologiques. Premièrement, une certaine « pression de publication » s'exercerait sur les chercheurs dans la mesure où l'approche en cas unique dans une perspective de neuropsychologie cognitive ne serait plus au goût du jour. Deuxièmement, ce type d'approche nécessiterait la mise en place de collaborations entre chercheurs et cliniciens en raison de la complexité croissante de nos connaissances sur le cerveau. Troisièmement, ce désintérêt pourrait s'expliquer par l'attrait de moins en moins important des étudiants pour la neuropsychologie au profit du domaine des neurosciences. En suivant cette voie, les étudiants n'auraient pas l'opportunité d'étudier et d'acquérir des modèles solides de psychologie leur permettant par la suite, s'ils le désirent, de mieux comprendre le fonctionnement psychologique avec une approche neuroscientifique. Enfin, la composante de neuroimagerie jouerait un rôle non-négligeable dans ce désintérêt en ce qu'elle aurait une influence sur l'attribution des bourses et des financements grâce à son aspect " novateur » et " blingbling ».

Le rôle de la neuropsychologie cognitive et de la neuroimagerie dans la compréhension du fonctionnement psychologique

Neuropsychologie cognitive et neuroimagerie sont étroitement liées et permettent de contribuer à la compréhension du fonctionnement psychologique normal ainsi qu'à la localisation des processus psychologiques dans le cerveau. L'apport de la neuroimagerie à la neuropsychologie cognitive a été critiqué. Ainsi, des chercheurs pensent que la localisation d'un processus psychologique dans le cerveau n'aiderait pas à comprendre son fonctionnement. La grande majorité des études en neuroimagerie viserait donc à localiser plutôt qu'à informer sur le fonctionnement d'un modèle psychologique. La neuropsychologie cognitive et la neuroimagerie ont également été défendues en regard de ce qu'elles peuvent apporter à la compréhension des processus psychologiques. Ainsi, certains auteurs suggèrent que la neuropsychologie cognitive, qui viserait à comprendre le fonctionnement psychologique, ne serait pas « morte » et devrait s'étendre à d'autres domaines que le langage 
ou la sémantique [11]. D’autre part, selon Mara Mather [12], la neuroimagerie aurait tout de même permis de mieux comprendre le fonctionnement de certains processus cognitifs et ne serait donc pas totalement désuète. Enfin, plusieurs chercheurs partagent une vision intégrative et suggèrent que les neurosciences, investiguées grâce à la neuroimagerie, auraient besoin de l'étude des processus comportementaux afin d'éviter de tomber dans un biais réductionniste [13].

\section{Changer nos approches et nos méthodes d'évaluation ? Une nécessité}

Plutôt que de trancher entre ces deux approches, il convient de donner une place plus importante aux analyses psychologiques en neuropsychologie. Notre fonctionnement psychologique au quotidien est très complexe et cette complexité doit être prise en compte. Cette prise en compte de la complexité du comportement passe par l'utilisation de protocoles de recherche riches et qui se rapprochent le plus possible de notre fonctionnement quotidien. Martial Van der Linden dénonce ainsi la pauvreté des designs utilisés dans certains domaines de recherche ayant recours à des approches en laboratoire et à des stimuli isolés (mots, images, chiffres...). En clinique, il faut également défendre une approche intégrative qui ne réduit pas les problèmes des patients à leurs lésions et qui prend en compte des facteurs d'identité et de motivation.

\section{L'exemple de la mémoire épisodique}

Martial Van der Linden illustre cette approche avec un paradigme auquel il a récemment contribué et qui vise à mieux comprendre le fonctionnement de la mémoire pour des événements personnellement vécus. La mémoire épisodique n'échappe pas aux critiques faites à la recherche et est, la majorité du temps, étudiée en laboratoire sans que cela reflète les processus mnésiques tels qu'ils sont mis en place dans la vie quotidienne [10]. 
Afin de mieux modéliser le fonctionnement de la mémoire épisodique, nous devons adapter notre vision de celle-ci aux modèles théoriques les plus récents (par exemple, le modèle de Conway [14]). Selon celui-ci, en segmentant le flux continu d'informations auquel nous sommes confrontés, nous créerions des souvenirs épisodiques formés à partir d'un moment psychologique de réalisation d'un but à court terme. La plupart de ces souvenirs ne demeureraient plus accessible intentionnellement après 4 ou 5 jours à moins que ceux-ci ne soient pertinents vis à vis de notre identité, de nos événements personnels ou de nos buts à long terme. Autrement dit, nous récupérerions en mémoire les aspects d'un événement qui correspondent à la conception que nous avons de nous-même ou du monde environnant. Afin de considérer un souvenir épisodique comme tel, nous réfléchirions sur nos propres états mentaux et nous disposerions de sentiments d'agentivité et de propriété personnelle qui nous permettraient de prendre conscience de nos propres souvenirs. En se basant sur ce modèle, Martial Van der Linden et son équipe ont utilisé des appareils photos portatifs pour étudier comment nous segmentions ce flux continu d'informations en différents souvenirs et comment ces souvenirs résistaient à l'oubli après des délais de rétention variant jusqu'à un mois [15].

\section{Perspectives}

Cette vision de la mémoire épisodique souligne l'implication que ce type de nouveau protocole et de modèle théorique peut avoir pour l'avenir de la recherche et de la clinique. Ce modèle théorique et ce type de design permettraient la mise en place d'une évaluation de l'expérience phénoménologique qui pourrait être appliquée en recherche aussi bien dans les études de cas uniques que dans les études de groupe. En clinique, cette approche induirait des changements dans la sémiologie ainsi que dans les outils diagnostics utilisés. Martial Van der Linden ne voit toutefois pas l'intérêt que confèrerait l'approche en neuroimagerie à la compréhension de ce type de modèle.

Malgré la richesse de ce débat, toutes les facettes de la relation entre neuropsychologie et neuroimagerie n'ont, bien sûr, pas pu être abordées. D'une part, il convient d'insister sur le rôle de plus en plus important des biomarqueurs dans les diagnostics des démences et des 
dégénérescences neuropathologiques. A l'avenir, ces biomarqueurs vont vraisemblablement être utilisés de façon de plus en plus précoce et la neuropsychologie devra, d'une façon ou d'une autre, s'en accommoder. D'autre part, les techniques les plus récentes d'analyse de neuroimagerie (connectivité fonctionnelle et analyses multivariées) devraient permettre, à l'avenir, de mieux modéliser le fonctionnement de la mémoire épisodique tel qu'il est défendu par Conway.

\section{Conclusion}

En raison de la difficulté à modéliser et à interpréter les réponses cérébrales qui lui sont associées (par exemple le signal BOLD), l'implication de la neuroimagerie dans la compréhension de la cognition normale semble demeurer matière à discussion. Malgré leurs avis divergents sur la question, les deux orateurs s'accordent pour dénoncer la dérive « neuroculturelle " (l'investigation des substrats neuronaux de tous les phénomènes possibles et inimaginables) à laquelle nous sommes confrontés dans notre domaine. En outre, la neuropsychologie doit s'inscrire dans une approche intégrative qui prend en compte divers facteurs autres que cognitifs ou lésionnels. Cette approche passe par l'utilisation de modèles psychologiques valides et par l'emploi de protocoles de recherches novateurs qui nécessitent la mise en place de processus mentaux qui se rapprochent le plus possible de ceux que nous mettons en place dans notre vie quotidienne. Cet appel au renouveau de la neuropsychologie s'inscrit dans l'évolution de notre époque et souligne l'importance de nous adapter aux nouvelles méthodes qui sont à notre disposition. Cette vision de la neuropsychologie cognitive tournée vers l'avenir introduisit à merveille la conférence de clôture de cette journée d'anniversaire : "La neuropsychologie à l'ère du numérique et des nouvelles technologies".

\section{Références}

[1] Michel D, Laurent B, Foyatier $\mathrm{N}$ et al. Left paramedian thalamic infarct. Memory and language study. Rev Neurol (Paris) 1982; $138:$ 533-50. 
[2] Schott B, Laurent B, Mauguière F. Thalamic pain: critical study of 43 cases. Rev Neurol (Paris) 1986;142:308-15.

[3] Milner B, Corkin S, Teuber H-L. Further analysis of the hippocampal amnesic syndrome: 14-year follow-up study of H.M. Neuropsychologia 1968;6:215-34.

[4] Dehaene S, Pegado F, Braga LW et al. L'impact de l'apprentissage de la lecture sur le cerveau. Med Sci (Paris) 2011;27:236-8.

[5] Barbeau EJ, Taylor MJ, Regis J et al. Spatio temporal Dynamics of Face Recognition. Cereb Cortex 2008;18:997-1009.

[6] Fauchon C, Faillenot I, Perrin AM et al. Does an observer's empathy influence my pain? Effect of perceived empathetic or unempathetic support on a pain test. Eur J Neurosci 2017;46:2629-37.

[7] Ingalhalikar M, Smith A, Parker D et al. Sex differences in the structural connectome of the human brain. Proc Natl Acad Sci 2014;111:823-8.

[8] Wiech K, Farias M, Kahane G et al. An fMRI study measuring analgesia enhanced by religion as a belief system. Pain 2008;139:467-76.

[9] Bianchi-Demicheli F, Cojan Y et al. Neural bases of hypoactive sexual desire disorder in women: an event-related FMRI study. J Sex Med 2011;8:2546-59.

[10] Van der Linden M. Pour une neuropsychologie clinique intégrative et centrée sur la vie quotidienne. Rev neuropsychol. sous presse.

[11] Coltheart M. The assumptions of cognitive neuropsychology: Reflections on Caramazza (1984, 1986). Cogn Neuropsychol 2017:1-6.

[12] Mather M, Cacioppo JT, Kanwisher N. How fMRI can inform cognitive theories. Perspect Psychol Sci J Assoc Psychol Sci 2013;8:108-13.

[13] Krakauer JW, Ghazanfar AA, Gomez-Marin A et al. Neuroscience Needs Behavior: Correcting a Reductionist Bias. Neuron 2017;93:480-90.

[14] Conway MA. Episodic memories. Neuropsychologia 2009; 47 : 2305-13.

[15] Jeunehomme O, Folville A, Stawarczyk D et al. Temporal compression in episodic memory for real-life events. Memory. sous presse. 